\title{
Assessment of dietary intake: NuGO symposium report
}

\author{
Linda Penn • Heiner Boeing • Carol J. Boushey • \\ Lars Ove Dragsted · Jim Kaput • Augustin Scalbert • \\ Ailsa A. Welch · John C. Mathers
}

Received: 22 December 2009/Accepted: 13 April 2010/Published online: 27 April 2010

(C) The Author(s) 2010. This article is published with open access at Springerlink.com

\begin{abstract}
Advances in genomics science and associated bioinformatics and technology mean that excellent tools are available for characterising human genotypes. At the same time, approaches for characterising individual phenotypes are developing rapidly. In contrast, there has been much less investment in novel methodology for measuring dietary exposures so that there is now a significant gap in the toolkit for those investigating how diet interacts with
\end{abstract}

L. Penn $(\bowtie) \cdot$ J. C. Mathers

Human Nutrition Research Centre,

Institute for Ageing and Health, Newcastle University,

Newcastle upon Tyne NE24HH, UK

e-mail: 1.d.penn@ncl.ac.uk

\section{H. Boeing}

Department of Epidemiology,

German Institute of Human Nutrition,

Potsdam-Rehbrücke, Germany

\section{J. Boushey}

Department of Foods and Nutrition, Purdue University, 700 W State St, West Lafayette, USA

L. O. Dragsted

Faculty of Life Sciences, Department of Human Nutrition,

University of Copenhagen, Copenhagen, Denmark

J. Kaput

Division of Personalized Nutrition and Medicine, National Center for Toxicological Research/FDA, Jefferson, USA

\section{A. Scalbert}

UMR 1019, Unité de Nutrition Humaine, INRA,

63122 Sainy-Genès-Champanelle, France

A. A. Welch

Diet and Health Group, Medical School,

University of East Anglia, Norwich NR47TJ, UK genotype to determine phenotype. This symposium reviewed the strengths and limitations of current tools used in assessment of dietary intake and the potential to improve these tools through, for example, the use of statistical techniques that combine information from different sources (such as modelling and calibration methods) to ameliorate measurement error and to provide validity checks. Speakers examined the use of approaches based on technologies such as mobile 'phones, digital cameras and Web-based systems which offer the potential for more acceptable (for study participants) and less laborious (for researchers and participants) routes to more robust data collection. In addition, the application of omics, especially metabolomics, tools to biofluids to identify new biomarkers of intake offers great potential to provide objective measures of food consumption with the advantage that data may be collected in forms that can be integrated readily with other high throughput (nutrigenomic) technologies.

Keywords Dietary intake - Assessment $\cdot$ Nutrigenomics · Phenotype $\cdot$ Exposure

\section{Introduction}

This symposium aimed to review the state-of-the-art in assessment of habitual dietary intake (exposure), including the strengths and limitations of conventional biomarkerbased approaches and to survey emerging "omics" based, and other, technologies for assessing dietary exposure. An individual's phenotype is the result of complex interaction between their genotype and environmental exposure. Dietary intake is a major contributor to environmental exposure. Over the past decade, the use of high throughput 'omics' technologies and other powerful new analytical 
methods in molecular biology have led to enhanced understanding of the genome and metabolic pathways. Genome wide association studies (GWAS) have facilitated the identification of common genetic factors that influence health and disease, and array-based gene expression profiles, proteomics and metabolomics have led to a comprehensive description of phenotype at a molecular level. Such approaches are transforming biomedical research. However, dietary assessment methodology is much less advanced. Fortunately, there is now a recognised need to reconsider the complex and difficult issue of measuring what people eat to address this gap and so contribute to further understanding of the relationship between food and health.

Environmental exposures, including diet, interact with an individual's genetic make-up to modulate phenotype and can be summarised by the equation:

Phenotype $=\sum[($ environmental exposure $) *$ genotype $]$.

In dietary assessment, we usually seek to determine current, or recent, habitual dietary intake, although dietary experiences from in utero to the time of investigation contribute to the total environmental exposure. With the rise in prevalence of nutrition-related chronic diseases, there is a greater appreciation of the long-term effects of diet on health. The use of new omics technologies has greatly improved our understanding of genotype and phenotype, but assessment of dietary intake has not advanced at the same rate. Dietary assessment is recognised as a critical, and complex, exposure variable that requires accurate assessment to provide reliable research outcomes, but current dietary assessment techniques may be subject to greater or lesser degree of error and bias [1-3] and present particular challenges for some population groups [4]. Improved methodologies and modern technologies may be used to address these shortcomings.

The symposium reported here analysed the strengths and limitations of the current methodology and presented potential new solutions for improved dietary assessment. The symposium was chaired by John Mathers (Newcastle University, UK) who introduced the topic. He dedicated the symposium to the memory of Professor Sheila Bingham, former Director of the MRC Centre for Nutritional Epidemiology in Cancer Prevention and Survival (Cambridge, UK), who had been a pioneer in developing better methods of dietary intake assessment.

Measuring what people eat is challenging because of the complexity, diversity and temporal heterogeneity of foods and food intake patterns. As a consequence, the commonly used tools which rely on participant reporting of intake e.g. diet diaries, recall methods and food frequency questionnaires (FFQ) have significant limitations. Dietary assessment terminology is defined in Table 1.

Newer research approaches focus on:

1. The use of modern technology and communication methods such as mobile 'phones, digital cameras and Web-based systems for more accurate and less arduous data collection;

2. The use of statistical techniques that combine information from different sources (such as modelling and calibration methods) to ameliorate measurement error and provide validity checks;

3. The application of omics tools to biofluids to identify new biomarkers of intake that will provide objective measures of food intake with the advantage of data collection in forms that can be integrated readily with other high throughput (nutrigenomic) technologies.

\section{Difficulties with dietary assessment methods and potential solutions}

Ailsa Welch (University of East Anglia, Norwich, UK) presented an overview of the strengths and limitations of current tools for assessing dietary intake, with potential solutions. Ideal dietary assessment methods would be quick, inexpensive, and easy to use and would give precise and accurate estimates of intakes of foods, nutrients, bioactive compounds, additives and contaminants, with minimal measurement error. However, dietary self-report instruments are subject to mis-reporting and errors and bias in data collection as well as errors in data processing: these include social desirability bias (where respondents report intakes in a manner consistent with perceived social norms rather than actual intake) and body mass index (BMI)related reporting bias (where under reporting is related to obesity). These biases affect research validity. The resources involved in collection and processing of data from the investigator perspective (the researcher burden) and from the research participant perspective (the respondent burden) are considerable, and data from different selfreport dietary assessment methods lead to differences in estimates of intake. To convert information on food intake to quantitative measures of intakes of energy, nutrients or other food constituents require both consideration of portion sizes, for example through food atlas photographs or weighed records, which adds to the data collection burden, and the use of food composition databases which increases the potential for further data errors.

The food supply is becoming more complex with increasing numbers of foods, especially processed foods, appearing in the market. Alterations in agronomic practices 
Table 1 Terminology in dietary assessment

\begin{tabular}{|c|c|}
\hline Food/diet diary & $\begin{array}{l}\text { A list (description) of foods eaten and drinks consumed } \\
\text { Usually includes an estimate of portion size in household measures } \\
\text { (e.g. slice of bread, cup of tea) } \\
\text { Written at the time of consumption }\end{array}$ \\
\hline Weighed food diary & $\begin{array}{l}\text { A list (description) of foods eaten and weight of each food before } \\
\text { (and remains after) consumption } \\
\text { Written at the time of consumption }\end{array}$ \\
\hline 24-h recall & $\begin{array}{l}\text { A list (description from memory) of foods consumed over the previous } 24 \mathrm{~h} \\
\text { Usually includes an estimate of portion size in household measures } \\
\text { (e.g. slice of bread, cup of tea) }\end{array}$ \\
\hline 'Multi-pass' 24-h recall & $\begin{array}{l}\text { A quick list (description from memory) of foods consumed over the previous } 24 \mathrm{~h} \\
\text { Usually includes an estimate of portion size in household measures (e.g. slice of bread) } \\
\text { Followed by questioning about the foods remembered to add detail } \\
\text { (e.g. type of spread on bread, type of milk in tea) }\end{array}$ \\
\hline Food frequency questionnaire & $\begin{array}{l}\text { A list of foods (marked from memory) to give an indication of the typical } \\
\text { frequency of consumption and amount consumed over a recent period of time (e.g. last year) }\end{array}$ \\
\hline Food intake patterns & $\begin{array}{l}\text { Variation in amount, type and frequency of food intake } \\
\text { a. The overall eating pattern e.g. Mediterranean eating pattern or Western-style eating pattern or } \\
\text { b. Some other characteristic features of intake e.g. skipping breakfast }\end{array}$ \\
\hline Basal metabolic rate (BMR) & $\begin{array}{l}\text { Energy expenditure due to vital bodily functions } \\
\text { Measured at rest and in thermoneutral environment } \\
\text { Largely determined by body size, especially fat-free mass } \\
\text { Typically } 60-75 \% \text { of total energy expenditure in developed countries }\end{array}$ \\
\hline Energy requirement & $\begin{array}{l}\text { Energy intake required to maintain equivalence between energy intake and energy expenditure } \\
\text { Can be expressed as a multiple of BMR }\end{array}$ \\
\hline $\begin{array}{l}\text { Cut offs for validation } \\
\text { of reported energy intake }\end{array}$ & $\begin{array}{l}\text { Based on the assumed equivalence of energy intake and expenditure at group level } \\
\text { and the confidence value below which the reported mean energy intake is unlikely } \\
\text { to represent valid data }\end{array}$ \\
\hline Group level & Determines probable degree of population bias in energy intake reporting \\
\hline Individual level & $\begin{array}{l}\text { Sometimes used to identify 'low energy reporters' where the energy intake is unlikely to be } \\
\text { valid. Ideally, should be used in conjunction with individual measure of energy expenditure } \\
\text { and assumes stable weight }\end{array}$ \\
\hline
\end{tabular}

and in food processing and storage methodologies can affect the chemical composition of foods. These factors pose challenges for identifying and characterising foods consumed by an individual. In addition, the increased complexity of dietary factors to be studied (bioactives, contaminants, additives) requires ever more sophisticated methodologies and databases.

Methods for identification of relative validity of dietary assessments include:

1. Comparison with other dietary assessment methods: for example food frequency questionnaires may be validated using 24-h recalls.

2. Use of predictor reference methods or cut-points for identifying mis-reporting where reported energy intake is compared with calculated energy expenditure using the principle of energy balance (e.g. Goldberg equations) [5].

3. 'Quantitative recovery' biomarkers: for example, 24-h urinary nitrogen excretion is determined largely by dietary intake of protein and can be used to estimate dietary protein intake for the previous 24-h period [6]. 4. 'Concentration biomarkers' may be suitable for relative ranking of intakes: for example plasma concentrations of highly unsaturated fatty acids can be used as biomarkers of relative intakes of dietary fatty acids [7].

The 7-day weighed food diary has been used commonly as the 'gold standard' for comparison with other dietary assessment methods and validity checks. In general, openended methods such as food diaries are regarded as more accurate than list-based methods e.g. FFQ [8, 9]. However, since 'person-specific reporting bias' indicates correlated within person measurement errors for self-report instruments, it is preferable to use objective biomarker methods for validation of self-report instruments where this is feasible. However, biomarker methods are also subject to errors including those due to inter-individual differences in metabolite responses related to individual gender and genotype, behavioural influences (e.g. impact of smoking 
on plasma vitamin $\mathrm{C}$ concentrations), non-linear associations of between exposure and biomarker concentrations and laboratory measurement error. Note, however, that dietary self-report and biomarker errors are independent.

\section{Web-based input, innovative 'dry' technology and statistical techniques}

Large-scale epidemiological studies have relied traditionally on FFQ methods of dietary assessment. Recently it has become apparent, from well-conducted validation studies with objective measurements and biomarkers of diet, that FFQ methods could pose a high degree of misclassification, thus masking existing relationships between diet and disease risk [10]. Heiner Boeing (German Institute of Human Nutrition, Potsdam-Rehbrücke, Germany) introduced the use of advanced and innovative dietary assessment methods and specialist statistical techniques associated with these new methods. By concept, FFQ, diary and 24-h recall methods all provide information on habitual dietary intake of an individual realised by the day to day consumption of foods (Illner et al. in press). The new efforts to improve the measurement of what people eat take advantage of the fact that information obtained from short-term standardised and computerised tools such as 24-h recalls is a better estimate of individual habitual intake than that obtained from FFQ methods. These short-term instruments can quantify dietary intake better than FFQs, but the problems of burden (for both researchers and participants), error, bias and cost outlined above remain relevant. Therefore, there is interest in the application of existing instruments via new communication strategies including delivery of traditional instruments through the internet [11]. In this new approach to dietary assessment, FFQs can also be used to provide covariate information about non-use and frequency of use of different foods. Computerised tools such as The ACASI "Audio computer-assisted interviewing"-FFQ [12] and the European food propensity questionnaire (FPQ) are now available. In addition, Webbased 24-h recalls have been developed by several groups in Europe and the United States such as the ASA24-SelfAdministered Interview 24-h recall [13, 14] and the (MXS) Web-based 24-h dietary recall. A Web-based 24-h recall "Etudé Nutrinet Santé" is being applied currently in a large epidemiological study in France. A new initiative the IMM "Interactive Multimedia"-recall has touch screen and audio functions to help data input [15]. The Innovation of Dietary Assessment Methods for Epidemiological Studies and Public Health (IDAMES) project aims to provide guidelines for the use of new methods of dietary assessment.
Digital imaging provides an innovative approach designed to improved dietary data collection. Examples of this are as follows:

- The Wellnavi Pocket-PC with digital camera and mobile phone for direct electronic data transfer to a dietician in the study centre [16];

- The Diet Mate ProPocket PC with integrated barcodescanner and associated Web-technology including the potential to establish an individualised database;

- Imaging and automatic estimation of food and portion sizes—see below for more details.

The increasing use of modern technologies prompted research on statistical methods that combine the information from short-term instruments such as 24-h recalls with longer-term instruments such as FFQ to improve dietary assessment in epidemiological studies. Such statistical methods are increasingly available as program packages available through the internet. In the multiple source method (MSM), frequency of use information from the FFQ is related to information from 24-h recall in respect of probability of use and amount eaten on each occasion using regression models. The portion size of a particular food that is recorded by an individual in a 24-h recall, can be compared against the probability that that individual would consume that food in that portion size based both on their own reported data from their individual FFQ and on the population probability for consumption of that particular food and portion size (https://nugo.dife.de/msm). To determine the likely relation to the population norms, individual variation in intake including allowance for covariate information (age, sex, food frequency) can be considered as well as population distributions. The European Food Consumption Validation (EFCOVAL-http:// www.efcoval.eu/) and Innovative Dietary Assessment Methods in Epidemiological Studies and Public Health (IDAMES—http://nugo.dife.de/twiki41/bin/view/IDAMES/) projects use this methodology $[17,18]$. There is potential to extend these statistical combination methods to incorporate the use of objective validation measures such as biomarkers.

\section{Digital imaging technologies}

Carol Boushey (Purdue University, USA) described her work on novel technologies for assessing dietary intake. The National Cancer Institute (NCI) and the National Heart Lung and Blood Institute (NHLBI) as part of the Exposure Biology Program within the National Institutes of Health (NIH) Genes, Environment, and Health Initiative (GEI) are addressing the challenges facing self-report methods including dietary assessment $[19,20]$. Carol Boushey's presentation focussed first on the Technology Assisted 
Dietary Assessment (TADA) project. This involves a novel food record method using a mobile device with an embedded camera to estimate daily food and nutrient intake among adolescents and adults [21].The mobile device provides a unique vehicle for collecting dietary information that reduces reporter burden. The method includes the use of digital image analysis for identification and quantification of food consumption based on images of the food items. Data from images obtained before and after food is consumed can be used to link the identified foods and amounts eaten with a food composition database [22]. In the design and testing phase, adults and adolescents participated in controlled meal studies (Six BL, Schap TE, Zhu FM, Mariappan A, Delp EJ, Ebert DS, Kerr DA, Boushey CJ: Evidence-based development of a mobile telephone food record. J Am Diet Assoc (in press)). The participants were asked to use a mobile phone camera to capture an image that included all foods and beverages, and a fiducial marker (an item of known size), before and after eating. The number of images per meal that participants took was counted and the use of the mobile phone food record (mpFR) was discussed in interactive sessions. Both age groups agreed that using the mpFR was easy, and repeated use allowed people to become more facile with the device e.g. the proportion needing to take only one image to capture foods and beverages either before or after increased significantly. The outcomes from these studies suggest that training requirements for users of the mpFR will be different across the lifespan.

In the TADA system, the food images and metadata are processed with image recognition technology that includes identification of food type and estimation of portion size by volume. The fiducial marker in the image is essential to allow colour correction for food image recognition and for estimation of food volume. Portion size is estimated in cubic centimetres and current databases, such as the Food and Nutrient Database for Dietary Studies (FNDDS), use weight measures. X-ray computerised microtomography (XMCT), 3D laser imaging, and other techniques are being used to measure food density (grams/cubic centimetres) to allow for conversion of portion estimates to a weight measure. Carol Boushey and her colleagues plan to validate the mpFR and imaging technology in free-living, individuals using doubly labelled water.

Modern 'dry' technology, photo-diaries, image recognition, direct Web input and improved statistical techniques will improve the collection of dietary data, reduce the researcher and respondent burden, and reduce the potential for data collection and input error. These methodological advances are expected to help address the need for greater precision in dietary assessment required to complement the step change in understanding of the molecular mechanisms through which dietary factors and genotype interact and the phenotypic consequences of such interactions. Nevertheless, a degree of subjectivity in selfreport measures remains. Therefore, analysis of biofluids both for objective validation of self-report instruments and to provide objective, although indirect, assessment of dietary intake is a promising area for research.

\section{Food chemistry, xenobiotic metabolism and dietary assessment: bioinformatics and metabolomics to decipher their complex links}

Augustin Scalbert (Unité de Nutrition Humaine, INRA, France) introduced biomarker approaches to dietary assessment. Several thousand molecules have been identified in foods and some of these metabolites are characteristic of a given food species or group of food species. After food ingestion, metabolites are found in the systemic circulation and tissues and are excreted in urine. Being characteristic of specific foods and diet, these metabolites provide objective measures of what people have eaten [23]. To date, the shortage of properly validated exposure biomarkers limits the use of biobanks of blood or urine to determine dietary exposure. The challenge, therefore, is to interrogate the wealth of information contained in the blood and urine metabolome to identify robust and specific markers for all foods commonly consumed.

Two different approaches can be used to identify such markers.

- First a hypothesis-driven (top down) approach where previous knowledge of food composition and of the metabolism of food constituents is used to identify putative markers of food intake. Their value as biomarkers is then evaluated by measuring correlations between their concentrations and the intake of the corresponding food sources.

- Second a data-driven (bottom up) approach where metabolome fingerprints are analysed in biofluids (urine or plasma) collected after consumption of a well-characterised diet and the characteristic metabolites identified.

For the hypothesis-driven approach, macronutrients and micronutrients, although well characterised in food composition databases, are too ubiquitous to be of value as biomarkers of specific food intake whereas other 'minor' non-nutrient compounds that have potential as specific biomarkers are not well characterised in food databases. These 'minor' compounds are numerous in foods-for example, in the tomato 869 metabolites including 70 flavonoids and 93 glycoalkaloids have been identified. Present sources of information about minor compound 
include the Dictionary of Natural Products, Dukes' Phytochemical and Ethnobotanical DB (http://www.ars-grin. gov/duke), USDA DB (http://www.nal.usda.gov) and KNApSAcK DB (http://kanaya.naist.jp/KNApSAcK). Augustin Scalbert's presentation illustrated this hypothesisdriven approach by focussing on polyphenols, one of the more diverse and widespread classes of food phytochemicals. A comprehensive database (Phenol-Explorer) describing all known polyphenols in foods has been built (http://www.phenol-explorer.eu), and an inventory of all known polyphenol metabolites formed in the body has been made. Associations between intake of polyphenolrich foods and some of these metabolites in urine samples of free-living subjects have been characterised. The next stage for this hypothesis-based approach is to build similar database resources for other minor food components such as carotenoids, glucosinolates, phytosterols and alkaloids. There are already a number of studies that link various minor compounds in biofluids to specific foods or food categories and, therefore, are indicative of specific food intake [23-25].

To illustrate the data-driven approach, Augustin Scalbert described a proof-of-principle metabolomics study that was carried out in his laboratory to identify markers of consumption of citrus fruit juices. Urine was collected from volunteers following consumption of orange or grapefruit juice and analysed using liquid chromatography timeof-flight mass spectrometry (LC-QTOF) to produce metabolome fingerprints. Principal component analysis (PCA) score plots were shown to discriminate clearly the consumption of orange and grapefruit juices. Characteristic urinary markers belonging to different classes of phytochemicals were identified using a new in-house phytochemical metabolite database.

The construction of databases containing major known metabolites characteristic of specific foods as described for polyphenols should help annotating the food metabolome. A common repository for annotated and non-annotated metabolites in biofluids should facilitate the identification of new specific markers of intake for the most frequently consumed foods.

\section{New technologies: metabolomics in experimental studies for biomarker development}

Lars Ove Dragsted (University of Copenhagen, Denmark) described experimental studies with tightly controlled dietary conditions using a data-driven approach to derive information on potential biomarkers of specific food components. Exploration of the metabolic profiles from such samples may identify markers relating to one of the following:
- It may be a valid exposure marker of a food i.e. a metabolite of a specific compound found in the food or in the food class,

- It may be an early effect marker related to the food i.e. an effect that often occurs following ingestion of the food item (typically a food-host interaction such as a microbial metabolite or a biochemical response), or

- It may be either i.e. an unusual diet-host interaction or even a chance finding.

In an experimental study using rats, Lars Ove Dragsted's team investigated the differential effects of apple and onion-based diets on metabolomic profiles in urine and plasma samples. Five different intervention diets were compared with a control diet. In the metabolome profiling analysis a metabolome standards mix was run alongside each batch for reference purposes. The basic procedure involved analysis of duplicate samples in random order using liquid chromatography time-of-flight mass spectrometry (LC-TOF) to obtain complex profiles that were compared with standards to select potential markers. After feeding the onion diet, some biomarkers in rat urine were increased and some were decreased. Of those metabolites that were increased, some were identified as unique to onion. Other metabolites were strongly increased or decreased in response to the onion products but were also present in the controls. One of these urinary metabolites is involved in steroid metabolism and further analyses showed increased concentration of plasma cholesterol and up regulation of the related hepatic pathway following the onion diet in rats. When the same basic procedure profile analysis was applied to rat urine in the apple diet study, potential specific markers for apple intake were identified. In addition plasma cholesterol concentration was lowered after apple feeding.

Lars Ove Dragsted then reported a dietary study with apples in human volunteers and the search for specific apple markers common to both humans and rats. Some, but not all, of the specific apple markers were found to be common between rats and humans and some of these common markers were previously unknown organic compounds. He reported analysis of 350 plasma samples from the Diet, Cancer and Health Cohort (DCH), a branch of the Danish EPIC study, for the unique biomarkers for onion and apple exposure identified through the experimental dietary studies described earlier. The preliminary analysis did not show more significant differences than expected based on chance between the individual plasma markers in people who subsequently developed colon cancer and those who did not. It is important to note that these cohort analyses were done on morning plasma samples and the tight homeostatic control of plasma may preclude identification of major differences in specific compounds present 
even when these would be apparent from urine analysis. However, subsequent analysis for complex pattern changes may lead to discriminative "combined markers" in plasma as well as in urine. At this stage, it would appear that urine is likely to be the more useful biofluid in the search for specific markers, especially when the sample represents a 24-h collection period.

In conclusion, the results from experimental studies, suggest that metabolome approaches show promise as a route to identify novel food intake markers that are specific to particular foods.

\section{Diet-related epigenomic marks}

To date most dietary assessment methods provide a snapshot of current or recent dietary intake. However, John Mathers (Newcastle University, UK) noted that it is probable that homeostatic mechanisms, which evolved to buffer the inevitable swings in food availability to which Homo sapiens (and its predecessors) has been exposed, ensure that short-term changes in food intake have little or no effect on health. Of course chronic exposures to extremes of dietary intake e.g. sustained under-nutrition which leads to depletion of body reserves and adverse effects on many body systems including immune function, or over-consumption of energy leading to obesity, have very obvious phenotypic sequelae. For other diet-related complex diseases, such as many cancers, cardiovascular disease, Alzheimer's disease, osteoporosis and type 2 diabetes (T2D), a direct link between exposure and outcome is less obvious. It seems probable that dietary exposures over long-time periods-years or decades - may be important in the aetiology of such diseases. In addition, in some cases the precipitating dietary exposure may have occurred at some time quite remote from the diagnosis of ill-health e.g. poor nutrition in utero may contribute to T2D risk many decades later. This poses particular challenges when attempting to provide data for the "environmental exposure" variable within the equation below:

Phenotype $=\sum[($ environmental exposure $) *$ genotype $]$.

John Mathers (Newcastle University, UK) introduced the potential use of epigenetic markers of long-term dietary exposure. Exploration of the impact of diet on epigenomic markings may offer a novel approach to the characterisation of long-term dietary exposure. Epigenomic markings include altered patterns of DNA methylation (addition of methyl groups to the $5^{\prime}$ position of cytosine residues usually when adjacent to a guanine residue $(\mathrm{CpG}))$ and of the posttranslation modification of histone tails by covalent addition of acetyl, methyl, phosphate and other groups. There is growing evidence that a range of dietary exposures provoke changes in epigenomics markings and, because such marks are "remembered" across cell divisions, they may constitute a chromatin-based record of the totality of eating patterns.

John Mathers described the four 'Rs' of nutritional epigenomics. Over time and through exposure to the environment, including diet, the genome 'receives' information which is then 'recorded' and 'remembered' in epigenetic marks. These changes can be 'revealed' as altered gene expression [26].

Nutritional epigenetics research is at an early stage and the evidence base is fragmentary. Answers are needed for many questions including:

- Which dietary components affect epigenetic markings and, therefore, for which an epigenomics-based approach is potentially fruitful?

- Which epigenetic loci are responsive to dietary exposures and are these influenced by particular dietary factors?

- At what stages during the life-course are particular epigenetic loci affected by diet?

- Does a given dietary exposure have the same, or different, effects at different ages or stages of the lifecourse?

- How durable are epigenetic marks induced e.g. during growth in utero?

- What are the most appropriate technologies for "reading", and bioinformatics approaches for "interpreting", epigenomics patterns?

In conclusion, epigenetic marks are a complex and rich source of information. They are influenced by many environmental factors, including dietary factors, and patterns of epigenetic marks may provide a history of individual environmental exposures. Epigenetic marks may be especially useful for assessing lifelong (dietary) exposures.

\section{Strategic view from USA of dietary and physical activity assessments}

In the concluding presentation of the symposium, Jim Kaput (National Center for Toxicological Research/FDA, USA) introduced the concept of personalised nutrition based on genomic analyses, evidence-based data and mechanisms, health and disease biomarkers, nutrient and activity measures and cost benefit analyses for implementation. Current research strategies rely principally on data and risk factors determined at a population level. A key question for nutrigenomics research is whether those population risk factors can be converted to individual risk factors and, if so, how? More likely, new research 
strategies will be required to develop risks factors for individuals with similar metabolic profiles (essentially metabolic groups) [27].

The experimental designs of genomic and omic research, with some notable exceptions, typically fail to assess nutrient intake (e.g. energy in) or physical activity (energy out) [27]. The United States Department of Agriculture (USDA) and the US Food and Drug Administration (FDA) hosted an interagency workshop co-sponsored by the FDA, NIH, and USDA that was held at the USDAARS Beltsville facilities in Spring 2009 (in preparation). The workshop aimed to assess the dietary assessment tools that are available for researchers, what is needed, and how to proceed to develop and deploy new assessment approaches. Some tools are commercially available, for example, Viocare's (http://www.viocare.com) VioWell individual assessment and action planning scheme. The VioWell assessment tools include the Internet MultiEthnic Food Intake System (IMFIS), a tool that will include ethnic specific foods. This web-based tool will have professional food pictures with different portion sizes and will also include measures of physical activity (e.g. pedometers and accelerometers). The development of these tools will provide components and modules for the nutritional phenotype database being developed through international collaborations (van Ommen et al. submitted).

\section{Concluding comments}

This symposium highlighted the importance of diet as a key factor which interacts with genotype to determine phenotype. It is clear that, in contrast with the rapid advances in development of tools for research on the genome and on metabolic systems and pathways, there has been a lag in addressing the issue of robust assessment of dietary intake. This symposium identified several important new developments in tools for measuring food intake including those which use low cost, widely available and accepted technologies such as mobile phones and the internet for faster, and less expensive, data collection. Because of the complex relationships that humans have with their diet, all recording of diet by study volunteers is subject to a greater or lesser degree of bias. The application of omics approaches, especially metabolomic methodologies, to biofluids (e.g. blood, urine or saliva) offers exciting new opportunities to obtain quantitative information of habitual dietary intake with much lower risk of subject bias. However, data validation is of particular importance in dietary assessment and it seems probable that for the most robust estimates of intake it may be prudent to use a combination of methods.
There is an urgent need to make greater investment (both intellectual and financial) in developing improved methods for assessment of dietary intake. Failure to do so will delay the development of understanding of the consequences for phenotype of interactions between nutrition and genotype and the application of this emerging knowledge for improved human health and wellbeing.

Acknowledgments The European Nutrigenomics Organisation (NuGO): linking genomics, nutrition and health research, is a European funded Network of Excellence. NuGO was founded in January 2004 and there are currently 23 NuGO partner organisations from 10 European countries. This symposium on assessment of dietary intake was organised by NuGO Work Package Protocols and Procedures and held on 1st September 2009 in Montecatini Terme, Italy, as part of NuGO week 2009. The views of the authors do not reflect those of the FDA. LOD was supported in part by a Grant from Nordforsk, the Nordic Council of Ministers, for the Nordic Centre of Excellence on Systems biology in controlled dietary interventions and cohort studies (SYSDIET).

Open Access This article is distributed under the terms of the Creative Commons Attribution Noncommercial License which permits any noncommercial use, distribution, and reproduction in any medium, provided the original author(s) and source are credited.

\section{References}

1. Kikunaga S, Tin T, Ishibashi G, Wang DH, Kira S (2007) The application of a handheld personal digital assistant with camera and mobile phone card (Wellnavi) to the general population in a dietary survey. J Nutr Sci Vitaminol 53(2):109-116

2. Moshfegh AJ, Rhodes DG, Baer DJ, Murayi T, Clemens JC, Rumpler WV, Paul DR, Sebastian RS, Kuczynski KJ, Ingwersen LA, Staples RC, Cleveland LE (2008) The US Department of Agriculture Automated Multiple-Pass Method reduces bias in the collection of energy intakes. Am J Clin Nutr 88(2):324-332

3. Tucker KL (2007) Assessment of usual dietary intake in population studies of gene-diet interaction. Nutr Metab Cardiovas Diseases 17(2):74-81

4. Adamson AJ, Collerton J, Davies K, Foster E, Jagger C, Stamp E, Mathers JC, Kirkwood T (2009) Newcastle 85 + Study Core Team. Nutrition in advanced age: dietary assessment in the Newcastle 85 + study. Eur J Clin Nutr 63(Suppl 1):S6-18

5. Goldberg GR, Black AE, Jebb SA, Cole TJ, Murgatroyd PR, Coward WA, Prentice AM (1991) Critical evaluation of energy intake data using fundamental principles of energy physiology: 1: derivation of cut-off limits to identify under-reporting. Eur J Clin Nutr 45:569-581

6. McKeown NM, Day NE, Welch AA, Runswick SA, Luben RN, Mulligan AA, McTaggart A, Bingham SA (2001) Use of biological markers to validate self-reported dietary intake in a random sample of the European Prospective Investigation into Cancer United Kingdom Norfolk cohort. Am J Clin Nutr 74(2): 188-196

7. Hodson L, Skeaff CM, Fielding BA (2008) Fatty acid composition of adipose tissue and blood in humans and its use as a biomarker of dietary intake. Prog Lipid Res 47(5):348-380

8. Gibson RS (2005) Principles of nutritional assessment. Oxford University Press, Oxford 
9. Welch AA, Benjamin C (2005) Dietary intake measurement I methodology, in encyclopedia of human nutrition. Elsevier, Oxford, pp 7-16

10. Schatzkin A, Kipnis V, Carroll RJ, Midthune D, Subar AF, Bingham S, Schoeller DA, Troiano RP, Freedman LS, Kipnis V et al (2003) A comparison of a food frequency questionnaire with a 24-h recall for use in an epidemiological cohort study: results from the biomarker-based Observing Protein and Energy Nutrition (OPEN) study.[see comment]. Int J Epidemiol 32(6):10541062

11. Ngo J, Engelen A, Molag M, Roesle J, Garcia-Segovia P, SerraMajem L, Engelen A, Molag M, Roesle J, Garcia-Segovia P et al (2009) A review of the use of information and communication technologies for dietary assessment. Br J Nutr 101(Suppl 2):S102-S112

12. Slattery ML, Murtaugh MA, Schumacher MC, Johnson J, Edwards S, Edwards R, Benson J, Tom-Orme L, Lanier AP, Slattery ML et al (2008) Development, implementation, and evaluation of a computerized self-administered diet history questionnaire for use in studies of American Indian and Alaskan native people. J Am Diet Assoc 108(1):101-109

13. National Cancer Institute US. Automated self-administered 24-h dietary recall (ASA24). 2009 [cited 12/09]; Available from: http://riskfactor.cancer.gov/tools/instruments/asa24/

14. Subar AF, Thompson FE, Potischman N, Forsyth BH, Buday R, Richards DMS, Hull SG, Guenther PM, Schatzkin A et al (2007) Formative research of a quick list for an automated self-administered 24-h dietary recall. J Am Dietetic Assoc 107(6):10021007

15. Zoellner J, Anderson J, Gould SM (2005) Comparative validation of a bilingual interactive multimedia dietary assessment tool. J Am Diet Assoc 105(8):1206-1214

16. Wang DH, Kogashiwa M, Ohta S, Kira S (2002) Validity and reliability of a dietary assessment method: the application of a digital camera with a mobile phone card attachment. J Nutr Sci Vitaminol 48(6):498-504
17. EFCOVAL. EFCOVAL project. European Community FP6 FOOD-CT-2006-022895 2006 [cited 12/09]; Available from: http://www.efcoval.eu/publications/EfcovalflyerA4layout.pdf

18. IDAMES. Innovation of dietary assessment methods for epidemiological studies and public health. 2007 [cited 12/09]; Available from: http://nugo.dife.de/twiki41/bin/view/IDAMES/

19. Boushey CJ, Kerr DA, Wright J, Lutes KD, Ebert DS, Delp EJ (2009) Use of technology in children's dietary assessment. Eur J Clin Nutr 63(Suppl 1):S50-S57

20. Winter J, Boushey CJ (2009) Workshop 1: use of technology in dietary assessment. Eur J Clin Nutr 63:S75-S77

21. Zhu L, Meier K (2008) Put schools on diet: health inequality and policy learning in fighting youth obesity. In paper presented at the annual meeting of the APSA, 2008

22. Mariappan A, Ruiz MB, Zhu F, Boushey CJ, Kerr DA, Ebert DS, Delp EJ (2009) Personal dietary assessment using mobile devices. Proceedings SPIE-the international society for optical engineering, 7246:72460Z, pp 1-12

23. Manach C, Hubert J, Llorach R, Scalbert A (2009) The complex links between dietary phytochemicals and human health deciphered by metabolomics. Mol Nutr Food Res 53:1303-1315

24. Ito H, Gonthier M-P, Manach C, Morand C, Mennen L, Rémésy C, Scalbert A (2005) Polyphenol levels in human urine after intake of six different polyphenol-rich beverages. $\mathrm{Br} \mathrm{J}$ Nutr 94:500-509

25. Pollard J, Wild CP, White KLM, Greenwood DC, Cade JE, Kirk SFL (2003) Comparison of plasma biomarkers with dietary assessment methods for fruit and vegetable intake. Eur J Clin Nutr 57(8):988-998

26. Mathers JC (2008) Session 2: personalised nutrition epigenomics: a basis for understanding individual differences? Proc Nutr Soc 67(4):390-394

27. Kaput J, Rodriguez RL (2006) Nutritional genomics: discovering the path to personalized nutrition. In: Kaput J, Rodriguez RL (eds) Wiley, New York 